\title{
Téoros
}

Revue de recherche en tourisme

\section{Le nouvel âge du tourisme stratégique}

De la différentiation des produits au positionnement de

l'innovation

\section{Pascal Tremblay}

Volume 17, numéro 3, automne 1998

Pratiques contemporaines de gestion dans le domaine du tourisme

URI : https://id.erudit.org/iderudit/1072160ar

DOI : https://doi.org/10.7202/1072160ar

Aller au sommaire du numéro

Éditeur(s)

Université du Québec à Montréal

ISSN

0712-8657 (imprimé)

1923-2705 (numérique)

Découvrir la revue

Citer cet article

Tremblay, P. (1998). Le nouvel âge du tourisme stratégique : de la différentiation des produits au positionnement de l'innovation. Téoros, 17(3), 11-17. https://doi.org/10.7202/1072160ar 


\section{twa..... \\ LE NOUVEL ÂGE \\ DU TOURISME STRATÉGIQUE \\ DE LA DIFFÉRENTIATION DES PRODUITS
AU POSITIONNEMENT DE L'INNOVATION}

\section{Pascal Tremblay}

La gestion du tourisme a, au cours des dernières années, suffisamnent progressé pour qu'on puisse comparer les difficultés émergentes avec celles dejjà identifiées dans d'autres champs de la planification stratégique. Bien que plusieurs problemes théoriques associés au développement de modeles de gestion stratégique aient éte identifiés ailleurs. ils présentent un intérêt spécial quand on les applique au tourisme. Ceci est, en partie, dâ au fait que l'on présente souvent le secteur des services comme particulier pour justifier l'élaboration de méthodes de gestion distinctes qui tiennent compte des caractéristiques fondamentales de l'offre de services'. Le fait que le champ du tourisme soit devenu, pour le meilleur et pour le pire, le signe avant-coureur de l' intemationalisation et d'un nouvel age du commerce et des relations socioculturelles constitue une raison supplémentaire pour explorer les fondations théoriques de modeles traditionnels de gestion stratégique. Selon des intervenants et des spécialistes en tourisme-et près de la thématique du présent numéro de Téoros - la capacité des divers ensembles or. ganisationnels (destination, industrie, firme...) à appliquer des méthodes de gestion qui permettent d'établir un avantage concurrentiel durable constitue un sujet qui, bien que longtemps peu développé, est maintenant reconnu comme crucial ${ }^{2}$.

\section{L'ÉVOLUTION DES PERSPECTIVES STRATÉGIQUES}

Pour évaluer les nombreux modèles de gestion stratégique appliqués au tourisme, il est utile de classifier sommairement les théories auxquelles ils se rattachent. Il devient possible, non seulement de démontrer que ces modèles ont rarement été spécifiques au tourisme, mais aussi de constater que la complexité du phénomène touristique et la grande variété de situations et de comportements associés au tourisme engendrent différentes interprétations dont découlent un grand nombre de prédictions théoriques contradictoires. Ainsi, le présent article adopte une approche évolutionniste qui se méfie des modèles présumant la convergence de structures et de comportements dans des systềmes complexes tels que celui du tou- risme. A tout le moins, cette approche constitue une mise en garde contre les généralisations prématurées relatives aux sources d'avantages concurrentiels dans le tourisme et dirige l'attention vers la grande variété de scénarios incompatibles qui tiennent pour acquis la convergence institutionnelle et organisationnelle.

\section{LES MODĖLES DE PLANIFICATION STRATÉGIQUE TRADITIONNELS}

Les modèles de planification stratégique traditionnels représentent la firme comme une entité capable de planifier sa position de marché (généralement définie par rapport à un choix de segment de marché ou de produit). La crédibilité de telles stratégies repose sur les contraintes imposées par la concurrence dans un contexte tech- nologique suffisamment bien établi et stable. Les travaux du Boston Consulting Group ${ }^{3}$ et de Porter (1980), par exemple, bien qu'ils supposent des caractéristiques différentes de l'environnement de la firme, identifient des stratégies génériques de positionnement du produit. Ces modèles constituent une première étape vers une représentation d'un environnement concurrentiel où, contrairement à la théorie économique néoclassique, la firme peut choisir sa stratégie marketing ; en fait, selon Porter, cela est même nécessaire à sa survie. Notamment, la firme doit établir une position stratégique par rapport au niveau de différentiation de ses produits (c'est-à-dire un mix cohérent prix-produit-choix de distribution), ainsi que le portefeuille des produits qu'elle pourra avantageusement offrir dans cet environnement, étant donné les positions occupées par ses concurrents, actuels et potentiels, et les possibles substituts. Ce type de modèle présume un contexte technologique donné et limite le domaine stratégique à l'identification de marchés cibles inexplorés ou à la domination industrielle par la minimisation des coûts ( $\alpha$ cosi leadership *). Cette représentation de la stratégie repose sur la conviction que l'environnement de la firme impose un nombre restreint de possibilités quand au développement et au positionnement de l'organisation, donnant place à une définition plutôt étroite de * l'avantage compétitif *.

\section{LE DÉVELOPPEMENT D'UNE APPROCHE ALTERNATIVE FONDÉE SUR LES COMPÉTENCES}

Au cours des dernières années, une approche différente de la gestion a adopté une vision davantage sophistiquée de la stra- 
tégie qui centre l'analyse sur le développement de compétences ${ }^{4}$ ou $\alpha$ capacités s. $^{5}$. Les bases de la compétitivité sont réorientées vers le développement et la différentiation des ressources déployées par la firme et son organisation dans le but d'établir une position concurrentielle tant au niveau de la technologie que du marché ${ }^{6}$. Dans ce contexte, la gestion stratégique permet la réorganisation de capacités ignorées par la concurrence ou simplement le développement de configurations technologiques radicalement nouvelles. On peut sûrement classifier l'approche de a stratégique $m$ dans la mesure où la firme n'est pas complètement dominée pas son environnement, mais il est clair que la composante $\alpha$ planification $\%$ prend un sens différent si elle exclut l'identification d'une vision déterministe des choix de comportements et de structures. Les capacités technologiques qui attirent l'attention de la firme sont diversifiées par rapport à leur accessibilité et au degré de difficulté liế à leur assimilation. Différentes organisations occupent des positions plus ou moins avantageuses pour approprier les techniques de production et de marketing nécessaires à leur survie. Cela veut dire qu'il ne peut y avoir de stratégie dominante s'appliquant également à toutes les organisations, même à l'intérieur d'une industrie donnée. La firme est donc représentée comme évaluant et contrastant diverses configurations de capacités qui évoluent au sein d'un complexe réseau technologique en mutation constante et dont la destination finale est inconnue.

Si les compétences techniques de la firme sont représentées par des agrégats de savoir-faire, on peut constater que ces derniers peuvent être classifiés comme plus ou moins similaires, selon la facilité avec laquelle ils peuvent être transférés et assimilés par différents individus ou organisations. Le degré de a similitude * (ou similarité) entre capacités et ressources représente donc le degré de parenté technologique entre divers éléments de savoirfaire et les activités qu'ils soutiennent. On peut aussi classifier diverses capacités ou compétences techniques selon les niveaux d'articulation et la complémentarité exigés quand on combine divers produits dans le but de permettre les échanges économiques. Le niveau de complémentarité (ou connexion, relatedness en anglais) Etablit une relation entre les composantes techniques qui doivent être combinées lors du choix des biens se prêtant à l'échange ou à la consommation. Ainsi, les connaissances requises dans les services hôteliers peuvent être caractérisées comme étant relativement plus \& similaires s aux capacités nécessaires dans d'autres secteurs de services basés sur l'hospitalité qu'à celles requises pour la production manufacturière. Par contre, un établissement comme un hôtel assemblera et devra gérer un nombre plus ou moins grand de ressources connexes au produit hôtelier. Il lui faudra donc combiner plusieurs compétences relevant de champs d'expertise variés. La sélection des compétences technologiques que la firme décide de développer devient l'élément central de sa stratégie, mais ne peut être établi à partir de simples déductions logiques basées sur une représentation complète de ses choix technologiques. Le succès dépendra éventuellement de la chance et des capacités imaginatives de la firme.

\section{L'APPROCHE COMPLÉMENTAIRE DE LA PERSPECTIVE ENTREPRENEURIALE}

Une approche complémentaire (ici décrite comme la théorie de la a firme entrepreneuriale $n$, mais en anglais normalement appelée " the learning firm *) permet d'élargir la notion de stratégie basée sur le développement de compétences, décrite ci-haut, tout en explorant un nombre de stratégies génériques liées aux changements qui surviennent dans l'environnement de la firme. Cette approche évolutionniste de la firme et de l'organisation industrielle établit un lien entre la capacité d'apprentissage d'un système et l'accumulation de compétences stratégiques de type entrepreneurial. Les décisions stratégiques de la firme sont vouées soit à $l^{\prime}$ adaptation aux circonstances imprévues auxquelles elle est soumise dans un environnement turbulent, soit au développement de nouvelles configurations de technologies et de produits, qui ont pour effet de transformer cet environnement et de changer les règles du jeu. Cette approche est véritablement évolutionniste puisqu'elle souligne le rôle de la diversité stratégique plutôt que celui de la convergence structurelle comme facteur explicatif de la stabilité relative des systèmes organisationnels et industriels complexes, tels que celui du tourisme. Aussi, l'approche n'est pas déterministe, car la firme doit choisir entre plusieurs motivations stratégiques et imaginer les répercussions de ses choix sur sa position concurrentielle future. En d'autres mots, la firme doit interpréter son environnement et choisir une stratégie d'apprentissage qui suppor- tera la création et le développement de compétences qui assureront sa survie dans un avenir incertain.

\section{ALTERNATIVES STRATÉGIQUES POUR LA FIRME ENTREPRENEURIALE}

Cette section explore deux types de choix stratégiques auxquels la firme doit faire face quand les preneurs de décision reconnaissent les limites de leur rationalité et, surtout, envisagent la possibilité d'avoir des répercussions sur leur environnement ${ }^{7}$. Le but de cet exercice est de démontrer qu'une approche évolutionniste suggère non pas des recettes de succès ou des positions idéales pour la firme, mais établit plutôt que la viabilité organisationnelle repose sur la compétence à questionner et à imaginer l'évolution et la division des connaissances dans un champ industriel donné. Le niveau de compétence constitue une forme de méta-stratégie, procurant à l'entrepreneur une ultime source de vision stratégique : l'habileté de choisir entre diverses méthodes de croissance technologique. Ces choix sont particulièrement appropriés pour l'analyse du tourisme et des défis organisationnels auxquels font face les firmes qui opèrent dans un environnement en rapide évolution. Un principe fondamental dérivé de l'analyse économique peut servir de base à une justification sommaire des choix ${ }^{8}$. Le principe de « coût d'opportunité " peut s'appliquer à l'accumulation des connaissances. Toute activité productive, y compris l'apprentissage, nécessite l'exploitation de ressources qui pourraient être redéployées vers des utilisations alternatives. Le principe s'étend donc aux choix des méthodes d'apprentissage et des types de connaissances.

\section{LE RO̊LE DU DÉVELOPPEMENT DES PROCESSUS TECHNOLOGIQUUES}

La firme doit faire une choix : soit développer les connaissances des processus technologiques (process technologies ou process innovations), soit accumuler ou exploiter les connaissances du produit et du marché. Les deux types d'apprentissage sont très différents. Le premier suggère une stratégie de développement centrée sur une production rapide des connaissances de base du processus de production, afin d'abaisser le coût de production et d'ac- 
quérir une position dominante dans le développement des technologies de base (avec l'effet possible de générer ultérieurement des nouveaux produits ou des applications basées sur ces processus fondamentaux). Ce type de stratégie repose sur une organisation des compétences différente de celle nécessaire au développement de connaissances touchant l'évolution des marchés et des préférences ${ }^{9}$. L'innovation et le développer de produits requièrent une architecture et une culture organisationnelle differentes, car l'attention se porte vers la modularité des types de connaissances et la flexibilité à ajuster et à prédire les goûts et les motivations des consommateurs ${ }^{10}$. L'intérêt principal pour développer des principes de gestion stratégique c'est que l'organisation tendra à se spécialiser dans son approche à l'innovation et qu'il sera possible d'observer, à l'intérieur d'une population de firmes, un niveau considérable de divergence stratégique et de complémentarité.

Le second type de choix reflète le cout d'opportunité associé à tout investissement dans un processus de recherche et d'apprentissage. Conformément aux travaux de G.B. Richardson (1972), la coordination des compétences nécessaires aux activités de production et de consommation exige que certaines des connaissances essentielles soient développées à l'intérieur de la firme et que d'autres soient acquises à l'extérieur. On considère que l'achat ou l'acquisition par contrat de connaissances techniques préalablement développées est préférable quand il s'agit de ressources suffisamment standardisées. L'échange et la diffusion de connaissances radicalement nouvelles font genéralement appel à la coopération inter-entreprise. Les compétences jouant un rôle stratégique peuvent générer des profits en autant qu'on arrive à les développer avant la concurrence. Une organisation ne peut dominer tous les champs relatifs à sa croissance technologique, mais doit choisir entre un certain nombre de possibilités. Tout exercice de création de savoir-faire doit évaluer la position relative de l'organisation vis-à-vis ses concurrents dans la course technologique.

Cette observation en elle-même suggère qu'il faut maintenir des liens coopératifs avec les fournisseurs de produits complementaires et des substituts bien choisis afin d'évaluer la performance des concurrents dans leurs propres activités innovatrices. Les liens cooperratifs et les alliances jouent aussi un rôle dans la création de nouvel- les idées et la production de nouveaux concepts. L'interaction aboutit souvent à la comparaison de points de vue divergents, une condition nécessaire au développement d'idées originales. En fait, une grande diversité d'acteurs favorise la conception de nouvelles idees, en autant que ces acteurs soient suffisamment différents pour créer un certain niveau de tension, tout en étant suffisamment semblables pour arriver à communiquer ${ }^{11}$. Des liens trop rigides restreignent la flexibilité nécessaire à l'expérimentation, alors que l'absence de liens ou l'anonymat pourrait empêcher le niveau de communication requis pour la confrontation et l'évaluation d'un minimum d'idées. De plus, la plupart des innovations reposent sur la coopération de producteurs possédant des compétences différentes, et souvent complémentaires, qui doivent être conjointement modifiées. La capacité d'innover est donc favorisée par un niveau intermédiaire de structure (entre la hiérarchie trop rigide et l'anonymat du marché néo-classique) ; on pense notamment aux alliances stratégiques ou aux réseaux informels de communication (exigeant plus ou moins de collaboration effective) qui se forment spontanément dans la plupart des grappes industrielles.

La gestion de telles déviations technologiques requiert une certaine expérience liée à la rentabilité des différentes méthodes d'apprentissage dans divers milieux. Selon les principes de la méthodologie évolutionniste, le choix d'une stratégie en particulier tient compte du contexte spécifique de la firme et suggère qu'un système technologique est flexible et adaptable lorsqu'il invite et englobe un nombre suffisamment Glevé d'idées ou de points de vue différents. La création de réseaux permettant $d^{+} a c-$ céder à des nouvelles ressources et d'expérimenter des nouvelles configurations de ces ressources constitue une dimension critique de l'analyse stratégique moderne.

La présente section démontre clairement qu'une firme qui reconnaît que son environnement est incertain et changeant se doit d'évaluer un certain nombre de stratégies d'adaptation. Entre autres, elle doit considérer des champs de développement des connaissances alternatifs (ci-haut résumé par le contraste entre les connaissances du processus scientifique ou du marketing) et doit aussi décider s'il est préférable de faire l'apprentissage à l'interne ou à l'externe (les avantages de chaque méthode dépendent de la position préalable de la firme et de son environnement).

\section{PLANIFICATION STRATÉGIQUE EN TOURISME}

Les idées résumées dans les paragraphes qui précèdent ont eu des répercussions sur la gestion et le marketing touristiques, si ce $n^{3}$ est dans la pratique, tout au moins dans les rapports de recherche et publications scientifiques. Plusieurs auteurs ont promu l'application de la planification stratégique traditionnelle aux firmes cuvrant dans les secteurs du tourisme et de l'hếbergement ${ }^{12}$. L'approche stratégique a cependant été trop souvent considérée comme une panacée permettant de contrecarrer la volatilité des changements dans le milieu du tourisme, alors qu'elle n'est pas nécessairement en mesure de faire. Cette perspective permet d'énoncer une hypothèse implicite : le manque de planification est une cause majeure de l'instabilité du système touristique que les méthodes modernes de planification seraient en mesure de stabiliser. Ainsi, les firmes qui investissent dans la planification peuvent générer des rentes économiques.

Plus généralement, les promoteurs de la planification stratégique ont établi un lien théorique entre le niveau de maturité d'un secteur et les avantages de la planification. Si l'environnement est suffisamment stable et que la concentration horizontale s'intensifie, une planification détaillée est présentée comme essentielle à la survie. Ces plans sont spécifiques dans la mesure où différents segments de marché ou différentes positions technologiques doivent être approchés différemment des variables marketing traditionnelles. Ainsi, la firme se doit de choisir une position stratégique originale par rapport à la production ou à la distribution des ses produits (l'approche correspond à un « positionnement $»$ tel que suggéré par les multiples variations de l'analyse du Boston Consulting Group, du modèle de Shell ou du PIMS). Ou, si l'analyse de Porter (1980) est adoptée, la firme doit décider entre une stratégie de differentiation, de focus ou de a cost leadership $\%$, façonnant une sélection de ses produits, distincte des positions adoptées par ses concurrents. La planification stratégique peut donc être rationalisée par la turbulence dans l'environnement de la firme, en suggérant qu'une approche méthodique qui isole la firme de cette turbulence $1^{*}$ aidera à identifier des domaines concurrentiels où elle pourra occuper une niche bien définie. 


\section{LES APPROCHES PROACTIVES ET RÉACTIVES}

La planification stratégique est donc parfois interprétée comme proactive (en imposant la stabilité dans le milieu) et d'autre fois réactive (en s'isolant de la turbulence et en se concentrant sur une partie familière de l'environnement). En général, on parle de plus en plus de planification en fonction de l'évaluation des forces et des faiblesses internes de la firme touristique relativement à celles trouvées dans son milieu. Ce processus aide à formuler des objectifs pratiques qui permettent à l'organisation de mesurer sa performance relative et d'ajuster son analyse (Gap analysis). Ces recettes stratégiques; théoriquement, peuvent faire la différence entre le succès et l'échec dans le contexte touristique, mais il n'existe pas d'étude empirique liant leur adoption à des mesures de performance convaincantes ${ }^{13}$. On a identifié la capacité des groupes industriels (concentrés ou diversifiés) capables d'exploiter les synergies de marketing internes et de déployer leurs ressources dans de nouveaux marchés comme un facteur déterminant de l'avantage concurrentiel dans le tourisme international ${ }^{14}$. Si l'adoption précoce de méthodes rationnelles de gestion et de planification devait produire ce genre d'avantage concurrentiel, on devrait pouvoir observer et documenter le développement de groupes industriels qui dominent grâce à la concentration de leurs compétences organisationnelles (Lanfant poursuit cette ligne de pensée).

\section{LA PLANIFICATION STRATÉGIQUE ET LE NOUVEAU TOURISME}

Il est intéressant dans ce contexte d'évaluer le $\alpha$ nouveau tourisme $*$ en termes de ses prédictions et de l'approche à la gestion stratégique qu'il suppose. La thèse principale $\mathrm{d}^{7}$ Auliana Poon ${ }^{15}$ établit un contraste entre le $\alpha$ vieux tourisme $\%$ basé sur un modèle de gestion qu'elle qualifie de périmé, et le nouveau tourisme qui suggère une nouvelle philosophie de production et des paramètres différents pour la détermination de l'avantage concurrentiel. D'après elle, la philosophie soutenant la production touristique des années 1960 et 1970 mettait en valeur le tourisme de masse et la standardisation des produits et des techniques de distribution. Cela était en partie dû au contexte institutionnel et au besoin d'établir des structures et des normes dans le champ touristique qui, à l'époque, était en voie de développement et de reconnaissance. Cette approche aurait été remplacée depuis le années 1980 par un * tourisme nouveau $*$ dominé par la différentiation des produits, la segmentation des marchés et la conception de produits innovateurs.

La majeure partie de son argument ne constitue pas réellement une rupture avec les propositions traditionnelles de la gestion stratégique. La plupart des changements qu'elle identifie sont compatibles avec une stratégie de différentiation et d'exploitation des niches de marché (que Porter compare aux stratégies de domination par l'exploitation des économies d'échelle et la réduction des coûts). Ses propositions principales constituent plutôt l'observation d'un changement de types génériques de stratégie où le mix prix-produit-distribution est réévalué. Paradoxalement, l'analyse de Poon représente la mutation du vieux au nouveau tourisme comme étant en elle-même un phénomène de masse à portée globale. Non seulement les nombreuses références à un nouveau \& savoirfaire $n$ (ou a best practice $w$ ) suggèrentelles que la soi-disant nouvelle vague du tourisme est suffisamment répandue pour créer un nouveau contexte concurrentiel, mais aussi qu'il s'agit en soi de la nouvelle * clé du succès $\phi$.

Pourtant, interprétées dans le contexte de la firme entrepreneuriale, les transformations très rélles identifiées par Poon ne constituent qu'un changement de stratégie d'apprentissage où l'exploitation des connaissances de marché a remplacé le développement d'avantages associés aux techniques de base de production dans les fonctions touristiques devenues mûres. Ce changement découle directement du fait que, que dans plusieurs secteurs reliés aux services, il est plus profitable d'essayer d'exploiter les innovations relićes aux connaissances de produits et de marchés que les connaissances du processus technique de production ${ }^{16}$. Cela est dû au fait que les technologies génériques de processus sont plus faciles à repérer, à répliquer et à utiliser que les connaissances liées au marché qui sont spécifiques à l'emplacement et au contexte socioculturel. Il est donc difficile de protéger et d'approprier les rentes économiques associées aux innovations et aux progrès liés au processus car les concurrents tardent rarement à les imiter. Dans un tel contexte, les vertus de la planification ne proviennent pas de la méthode de décision et des paramètres qu'elle suggère, mais plutôt de la stimulation de l'imagination qu'elle crée et de l'élargissement des perspectives qu'elle entraîne $^{17}$. La planification est utile comme méthode et comme rituel, tant qu'elle demeure un outil d'exploration.

Il est donc dangereux d'interpréter l'apparition et la croissance de firmes flexibles et prospères comme le résultat d'une ruée vers l'or découlant d'une nouvelle philosophie « capable de générer des opportunités de profits sans fin ${ }^{18}$. L'impression qu'une majorité de firmes sont en mesure de créer des structures davantage flexibles et que cette nouvelle flexibilité a une incidence réelle sur leurs profits peut être trompeuse. Cette prédiction sousentend qu'il y a convergence de comportements et de structures dans le vaste secteur du tourisme. Mais malgré les tentatives répétées pour identifier des tendances lourdes concernant la structure industrielle et le comportement corporatif et organisationnel dans le système touristique, il demeure douteux que l'on assiste à une convergence gếnérale des stratégies d'intégration (suggérées par Lanfant) ou de flexibilité (identifiées par Poon). Tout au plus, est il intéressant de considérer l'observation de Cazes (1989) que nous sommes témoins d'un dualisme structurel reflétant la présence de mouvements vers l'intégration et la concentration (dans certains marchés ou certaines régions) et accompagné simultanément par la naissance et la croissance de petites et moyennes entreprises, souvent flexibles et différenciées, et exploitant des marchés cibles souvent volatiles.

\section{LA STRATÉGIE DU TOURISME : POSITIONNEMENT DE L'INNOVATION}

L'identification des sources de la diversité et de la volatilité du système touristique est nécessaire si l'on veut explorer la possibilité d'arriver à une approche stratégique qui soit applicable et suffisamment universelle pour la gestion et l"orientation stratégique en tourisme. Les écrits dans le domaine du tourisme décrivent deux types principaux de turbulence dans le milieu. Tout d'abord, il y a les facteurs externes tels que les changements des technologies de l' information et de la communication, qui sont développées en grande partie en-dehors du système touristique et qui peuvent générer des innovations radicales sur le plan du processus technologique ou de la création de produits. La source alternative majeure de turbulence dans le système touristique peut être con- 
sidêrée comme interne, étant donné son lien avec la motivation profonde des touristes. L'activité touristique est fondamentalement liée à la notion de changement et de nouveauté, que ce changement soit complémentaire au $\alpha$ temps de travail $*^{10}$, qu'il fasse partie d'une quéte d'authenticité ou de valeurs alternatives ${ }^{20}$, qu'il soit lié à la découverte de cultures et d'environnements différents, ou simplement qu'il reflète la recherche d'un climat contrastant avec le contexte quotidien. Le système touristique constitue un système de production volatile, en partie parce qu'il doit générer des expériences et des contextes plus ou moins différents pour des elients dont les attentes, en plus d'être très variées. $e^{e v o l u e n t}$ constamment ${ }^{21}$. Cela engendre nécessairement des innovations plus ou moins radicales, capables de déséquilibrer le système, et favorise le développement d'applications technologiques et d' institutions visant à balancer les besoins de flexibilité et d'intégration ${ }^{22}$.

Le développement des technologies génériques de l'information poursuit une trajectoire en grande partie indépendante des applications au tourisme. Ces dernières peuvent apparaître sous la forme de nouveaux processus technologiques à l'intérieur des fonctions touristiques traditionnelles (par exemple quand les innovations n'affectent pas directement le client qui ne perçoit que les changements dans les a caracteristiques marketing * du produit ; dans les prix, l'assemblage du produit, sa qualité ou sa distribution...). A d'autres occasions, elles rendent possible la génération de nouveaux produits (comme par exemple les programmes de \& frequent travellers * qui ont émergé comme produits dérivés de l'application de la télématique et des technologies parentes ${ }^{23}$ ). Mais l'apport le plus visible de ces nouvelles technologies a été d'affecter les liens entre les fonctions touristiques el de transformer leur frontières ainsi que leur importance relative ${ }^{24}$.

\section{DES TRANSFORMATIONS DIFFICILEMENT PRÉVISIBLES}

Ces transformations très imprévisibles et difficiles à approprier ont été la source de maintes craintes quand à leur approvisionnement. Si l'accès aux technologies devenait inégal, ou à tout le moins si certaines firmes mieux placées pouvaient profiter d'un avantage dans l'evaluation des incidences sur la structure et la nature des stratégies en tourisme, la structure orga- nisationnelle du tourisme pourrait en etre radicalement affectée ${ }^{25}$. En même temps, certains ont prophétisé la démocratisation de l'accès aux technologies de l'information pouvant éliminer les avantages dus aux économies d'échelles traditionnelles des grandes chaînes ou des groupes auvrant dans le tourisme. La balance de l'apport du développement de ces technologies sur la nature de l'avantage concurrentiel (au niveau de la firme dans le système touristique) dépend en grande partie des trajectoires technologiques particulières et de l'environnement institutionnel dans lequel elles se dessinent. Il est difficile d'identifier des tendances lourdes qui soient mondiales car les contextes culturels particuliers demeurent un facteur explicatif central des institutions et des organisations industrielles dans les divers espaces nationaux ${ }^{26}$,

\section{L'IMPÉRATIF DE LA RECHERCHE D'AVANTAGES CONCURRENTIELS}

Comme il n'existe pas de formule magique concernant l'adoption et la production de nouvelles technologies, il faut, dans la recherche d'avantages concurrentiels, expérimenter afin d'établir une vision du développement technologique et institutionnel. Alors que certains ont démontré que le développement de nouvelles technologies basées sur la standardisation des procédés et des produits pouvait donner lieu à des avantages concurrentiels soutenus $^{27}$, d'autres analystes ont suggéré que, dans les systèmes touristiques assez développés, les changements dans l'infrastructure de l'information pourraient promouvoir la création de produits à une plus petite échelle, ainsi que l'habileté à identifier de nouvelles niches et à se spécialiser dans de nouveaux segments de marche ${ }^{28}$. La création d'une « info-structure $\%$ commune permettrait en théorie aux petites et moyennes entreprises d'accéder aux ressources informationnelles, bien que leur positionnement à court terme demeure essentiellement réactif dans ce nouvel environnement ${ }^{25}$.

Ces scénarios plus ou moins divergents suggèrent que le développement d'une approche stratégique en tourisme doit reconnaître l'importance et la valeur de la diversité technologique dans un système turbulent. Il est possible de remplacer la notion de positionnement basée sur la différentiation des produits et des variables marketing (tels que décrits dans la gestion stratégique traditionnelle du Boston
Consulting Group et de Porter) par un positionnement relatif à l'accumulation stratégique des compétences. Une telle approche exige que les forces façonnant le processus d'innovation dans le processus touristique puissent être analysées, une hypothèse supportée par Lanquar (1976).

\section{STRATÉGIES ENTREPRENEURIALES ET PRODUIT TOURISTIQUE}

Cette section explore le lien entre l'objectif de développer une classification des positionnements stratégiques en tourisme basée sur l'innovation et les principes d'accumulation des connaissances décrits plus tôt. L'attention se porte, en particulier, sur les choix que la firme aeuvrant dans un système en evolution doit faire pour maintenir un avantage sur ses concurrents. La firme entrepreneuriale doit confronter diverses méthodes d'acquisition de compétences, principalement classifiées comme internes (acquises par la recherche et les investissements dans l'innovation au sein de l'entreprise) ou externes (par l'acquisition de technologies échangées avec des partenaires commerciaux ou partagées par des collaborateurs au sein de réseaux coopératifs). Les connaissances créées et acquises peuvent elles-mêmes être catégorisées en fonction de l'appartenance à l'innovation de produit ou à l'innovation de processus (ou procédé, process innovation en anglais), mais il est souvent difficile de faire la distinction et de faire des généralisations quant à leur importance relative dans le contexte du tourisme.

Lors de l'analyse de l'innovation dans le secteur des services, on dirige habituellement l'attention au développement de produits, parce que l'innovation liée au processus est en général considérée comme externe, peu contrôlable et difficile à approprier, à moins que des éléments tels que la réputation, les marques de commerce ou la culture d'entreprise propre soient développés ${ }^{30}$. L'orientation stratégique doit tout de même considérer la nécessité (et les coûts relatifs) du développement de nouveaux marchés et de canaux de distribution (souvent près des compétences centrales de la firme en tourisme) et le dépistage d'applications et d'opportunités nouvelles associées aux technologies existantes.

Pour une furme, la décision d'investir dans l'innovation de processus, de produit, ou une combinaison des deux, ne peut être 
évaluée indếpendamment de l'évolution technologique environnante. La complexité du produit touristique est souvent présentée comme le résultat d'un niveau de standardisation inadéquat des méthodes de production et d'un marketing inapproprié des caractéristiques des produits. Le tourisme constitue done un 4 quasi-produit $*^{31}$ car les spécifications sont mal établies et sujettes à des changements rapides. Cela permet une typologie de l'innovation en tourisme basée sur les changements dans le niveau de standardisation exigé par l'accumulation des nouvelles connaissances. Si le a produit tourisme $s$ est insuffisamment standardisé, les compétences nouvellement accumulées (par rapport au processus ou par rapport au marché-produit) peuvent soit renforcer et aider le processus de standardisation, soit le contrecarrer ${ }^{32}$.

Dans le premier cas, le produit générique * tourisme $*$ correspond de plus en plus à une configuration dont la composition est prévisible et la production planifiable. Cela est possible parce qu' on observe une convergence dans les processus de production et parce que les producteurs adoptent des positions différenciées (surtout au niveau des marchés et des produits). Il peut aussi y avoir convergence au niveau de la segmentation des marchés et de l'assemblage des produits, de telle sorte que les consommateurs adoptent des critères d'évaluation de plus en plus similaires. Dans ce dernier cas, l'avantage concurrentiel sera liế aux compétences particulières requises pour identifier rapidement des segments de marché rentables ou pour exploiter des synergies dans les procédés de production et de distribution.

L'innovation, volontairement ou non, peut aussi créer de nouvelles possibilités amenant une divergence dans la définition des marchés et des produits, ou dans les procédés de production et de distribution inhérents au secteur du tourisme. Dans ce dernier cas, la génération d'innovations opportunes, ou une flexibilité permettant de réagir et de s'ajuster aux innovations des concurrents, deviendrait l'avantage ultime.

\section{CONCLUSION}

On a suggéré une analyse de la stratégie reconnaissant la turbulence et la diversité structurelle du système touristique. Ces prémisses supposent que le processus de création des connaissances liées aux ser- vices et à l"assemblage des produits touristiques joue un rôle central dans la détermination de l'avantage concurrentiel. La volatilité associée aux motivations du touriste et à la nature du produit-expérience recherché favorise une attitude prudente par rapport à la prédiction de tendances organisationnelles et stratégiques. La diversité des apports technologiques et leur propension à être façonnés par le contexte socio-institutionnel suggèrent aussi qu'il est futile d'essayer d'établir des lois universelles concernant le succès de diverses stratégies de positionnement par rapport aux marchés et aux produits.

Il n'en demeure pas moins important d'essayer d'évaluer et de comprendre les forces déterminant la nature des mécanismes qui façonnent le changement technologique du système. Ceci permettrait entre autres d'améliorer notre compréhension du rôle de la diversité technologique afin d'assurer la flexibilité du système touristique et de minimiser les coûts socio-économiques associés à la vulnérabilité technologique et industrielle. A long terme, l'analyse de la répartition et de la diffusion des connaissances technologiques et de marketing du tourisme pourrait suggérer les institutions nécessaires aptes à supporter la capacité d'imagination du système touristique.

La constatation de la nature complexe et potentiellement éphémère du produit touristique donne lieu à un déplacement de la stratégie basée sur des choix technologiques relativement simples vers la métastratégie où le positionnement s'effectue par rapport à l'innovation et à la capacité d'exploiter avantageusement des synergies liées au développement des connaissances. Bien que situés à un niveau élevé de la hiérarchie stratégique, ces choix reflètent de façon critique le principe de coûts d'opportunité appliqué au déploiement des ressources nécessaires à touté activité innovatrice.

Ce positionnement met en relief les coûts associés à la capacité d'innover comparés à l'emprunt ou à l'échange d'idées développées par d'autres firmes. Le développement de conditions propices à l'innovation interne et a l'établissement de liens coopératifs requiert des ressources qu'on pourrait déployer différemment. On suggère done qu'il y a des avantages associés. a la spécialisation dans certains types de connaissances, et qu'il est relativement plus coûteux d'essayer de mélanger à l'aveuglette les processus innovateurs que de s'en tenir à un type stratégique. La suggestion de stratégies optimales basées sur l'appréciation des avantages et des désavantages de choix variés n'est cependant pas possible parce que cette appréciation repose sur un environnement volatile. De plus, les nouvelles connaissances qui affectent le processus de standardisation du produit et des technologies de production perturbent souvent l'ordre temporaire établi. La stratégie en tourisme est vouće à sa propre destruction.

Un comité de lecture a lu et accepté ce texte

\section{NOTES}

1 Thomas, 1978 .

2 Ritchie et Crouch, 1993.

3 Stern et Stalk, 1998.

4 Penrose, 1959.

5 Selon le langage de G.B. Richardson, 1972.

6 Foss et Knudsen, 1996 ; Hamel et Prahalad, 1994.

7 Cette section ne fait que résumer les points importants de l'approche. Pour une discussion exhaustive des choix stratégiques de la firme entrepreneuriale dans le contexte du tourisme, voir Tremblay, 1997 et 1998.

8 Voir Tremblay, 1997, pour un exposé complet.

9 Voir par exemple Dougherty, 1990 ; G.S. Day, 1994 ; Sanchez, 1995 ; Mowery, Oxley et Silverman, 1996

10 Langlois et Robertston, 1995.

11 Loasby, $1991: 56$.

12 Chon et Olsen, 1990 ; Flautre, 1986 ; Reichel, 1983 ; Schwaninger, 1986 ; Tribe, 1995 ; Wyckoff et Sasser, 1981

13 Athiyaman, 1995 ; Athiyaman et Robertson, 1995 : Gilbert et Kapur, 1990.

14 Lanfant, 1980 ; Simier, 1990.

$151988,1993,1994$; voir aussi Michaud, 1992. pour des idées similaires.

16 Lanquar, 1976 ; Michaud, 1992.

17 Moutinho, Rita et Curry, $1996: 109$.

18 Poon, $1994: 92$.

19 Krippendorf, 1986.

20 MacCannell, 1976.

21 Botterill, 1986 ; Goodall, Radburn et Stabler, 1988 ; Parinello, 1993 ; Urry, 1990.

22 Tremblay, 1997.

23 Mowlana et Smith, 1990.

24 Poon, 1993, chapitre 7 ; Hjalager, 1994.

25 Lanfant, 1980 ; Simier, 1990.

26 Houghton et Tremblay, 1994.

27 Par exemple : Lanfant, 1980, Cazes, 1989 et Simier, 1990.

28 Poon, 1993. 
29 Bressand et coll, 1989.

30 Lanquar, 1976 ; Casson, 1982.

31 * proto-commodity * dans le langage de Andersen, $1991: 134$.

32 Tushman et Anderson, 1986.

\section{RÉFÉRENCES}

Andersen, E.S. (1991), * Techno-Economic Paradigms as Typical Interfaces between Producers and Users *, Journal of Evolutionary Economics, vol. 1, p. 119-144.

Athiyaman, Adee (1995), \& The Interface of Tourism and Strategy Research : An Analysis *, Tourism Management, vol. 16, $\mathrm{n}^{0} 6, \mathrm{p}, 447-453$.

Athiyaman, Adee et R.W. Robertson (1995), * Strategic Planning in Large Tourism Firms : An Empirical Analysis ", Tourism Management, vol, 16, n०3, p, 199-205.

Botterill, T.D. (1986) * Dissatisfaction with a Construction of Satisfaction w, Annals of Tourism Research, vol. 13, p. 247-249.

Bressand, Albert, Catherine Distler, et Kalypso Nicolaildis (1989), a Networks at the Heart of the Service Economy $n$, sous la direction de Albert Bressand et Kalypso Nicolädis, Strategic Trends in Services - an Inquiry into the Global Service Economy, New York, Harper and Row, p. 17-32.

Casson. Mark C. (1982), \& Transaction Costs and the Theory of the Multinational Enterprise s, sous la direction de A.M. Rugman, New Theories of the Multinational Enterprise, London and Canberra, Croom Helm, p. 24-43.

Cazes, G. (1989), Le tourisme international mirage ou strategie d'avenir ?, Paris, Hatier.

Chon, Kye-Sung, et Michael D. Olsen (1990), * Applying the Strategic Management Process in the Management of Tourism Organizations \&, Tourism Management, septembre, p. 206-213.

Day, G.S. (1994), * Continuous Learning about Markets », California Management Review, vol. 94, p. 9-31.

Dougherty, D. (1990), * Understanding New Markets for New Products *, Strategic Management Journal, vol. 11, p. 59-78.

Flautre, Alain (1986), * La gestion de l'entreprise touristique au-dela de la gestion traditionnelle $*$, Teoros, vol. $5, \mathbf{n}^{0} 3$, p. $2-5$.

Foss, Nikolail J., et Christian Knudsen (dir.) (1996), Towards a Competence Theory of the Firm, London, Routledge.

Gilbert D.C., et R. Kapur (1990), * Strategic Marketing Planning and the Hotel Industry :, International Journal of Hospitality Management, vol. 9, $\mathrm{n}^{\circ} 1$, p. 27-43.

Goodall, B., M. Radburn, and M. Stabler (1988), * Market opportunity sets for tourism *, dans Geographical papers - lourism series, Reading, U.K., University of Reading.
Hamel, Gary, et A. Heene (dir.) (1994), Competence-Based Competition, Chichester, John Wiley and Sons.

Hjalager, A.-M. (1994), * Dynamic Innovation in the Tourism Industry $*$, sous la direction de C.P. Cooper et A. Lockwood, Progress in Tourism, Recreation and Hospitality Management, vol. 6, Chichester, John Wiley and sons, p. 197-224.

Houghton, John et Pascal Tremblay (1994), $\propto$ The Structure of Hospitality: A Cultural Explanation of Industrial Diversity $\%$, International Journal of Hospitality Management, vol. 13 , p. $327-346$.

Krippendorf, Jost (1986), « Tourism in the System of Industrial Society w, Annals of Tourism Research, vol. 13, p. 517-532.

Lanfant, Marie-Françoise $(1980)$ * Introduction: Tourism in the Process of Internationalization $\%$, International Social Science Journal, vol. 32, p. 14-43.

Lanquar, R. (1976), Notes pour une théorie du management de l'innovation dans l'entreprise touristique $\%$, Academie Internationale des Experts scientifiques du Tourisme, $\pi^{\circ}$ 76, p. 87-99.

Langlois, Richard N, et Paul L. Robertson (1995), Firms, Markets and Economic Change, London et New York, Routledge.

Loasby, Brian J. (1991), Equilibrium and Evolution = An Exploration of Connecting Principles in Economics, Manchester, Manchester University Press.

MacCannell, Dean (1976), The Tourist : a New Theory of the Leisure Class, London, Macmillan.

Michaud, Jean-Luc (1992), $\propto$ De l'économie de cueillette a l'industrie du futur $\%$, sous la direction de Jean-Luc Michaud, Tourismes - Chance pour l'économie - risque pour les societes?' Paris, Presses Universitaires de France, p. 17-26.

Moutinho, Luiz, Paulo Rita, et Bruce Curry (1996), Expert Systems in Tourism Marketing. London, Routledge.

Mowery, D.C., J.E. Oxley et B.S. Silverman (1996), "Strategic Alliances and Interfirm Knowledge Transfer $\%$, Strategic Management Journal, vol. 17, p. 77-91.

Mowlana, H., et G. Smith (1990), * Tourism, Telecommunications and Transnational Banking - A Framework for Policy Analysis w, Tourism Management, vol. 12, p. 315-324.

Parrinello, G.L. (1993), * Motivation and Anticipation in Post-Industrial Tourism *, Annals of Tourism Research, vol. 20, p. 233-249.

Penrose, Edith T. (1959), The Theory of the Growt of the Firm, Oxford, Basil Blackwell.

Poon, Auliana (1988), w Tourism and Information Technologies ${ }_{*}$. Amals of Tourism Research, vol. 15, p. 531-549.

Poon, Auliana (1993), Tourism, Techology and Competitive Strategies. Wallingford, U,K. CAB International.
Poon, Auliana (1994), \& The 'new tourism' revolution $*$, Tourism Management, vol. 15, $\mathrm{n}^{\circ}$ 2, p. $91-92$.

Porter, Michael E. (1980), Competitive Strategy : Techniques for Analyzing Industries and Comperitors, New York, The Free Press.

Reichel, Arie (1983), * Strategic Management : How to Apply it to Firms in the Hospitality Industry $m$, The Service Industries Journal, vol. 3. p. 329-343.

Richardson, George B. (1972), * The Organization of Industry s, Economic Joumal, $\mathrm{n}^{\circ} 82$, p. $883-896$.

Ritchie, J.R.B., et G.I. Crouch (1993), 4. Competitiveness in International Tourism - A Framework for Understanding and Analysis : Academie Internationale des Experts scientifiques du Tourisme, vol. 35, p. 1-42.

Sanchez, R, (1996), * Strategic Product Creation : Managing Interactions of Technology. Markets, and Organizations *, European Management Journal, vol. 14, p. 121-138.

Schwaninger, Markus (1986), * Strategic Business Management in Tourism $\cdots$, Tourism Management, juin, p. 74-85.

Simier, Paul (1990), * Le contrôle de la chaîne de distribution $\%$, Téoros, vol. $9, \mathrm{n}^{\circ} 3$, novembre, p. 3-6.

Stern, Carl W, et George Stalk, Jr (1998), Perspectives : the Best Strategic Thinking from the Boston Consulting Group, New York, John Wiley.

Thomas, Dan R.E. (1978), \& Strategy is Different in Service Businesses $\$$, Harvard Business Review, juillet-aoüt, p. 19-26.

Tremblay, Pascal (1997), \& Information and Coordination : The Economic Organization of Tourism *, thèse de doctorat, Faculty of Economics, University of Melbourne, Australia.

Tremblay, Pascal (1998), * The Economic Organisation of Tourism : A Network Approach *, Annals of Tourism Research, sous presse.

Tribe, John (1995), The Economics of Leisure and Tourism, Oxford, Butterworth-Heinemann.

Tushman, M.L., et P. Anderson (1986), * Technological Discontinuities and Organizational Environments $\%$, Administrative Science Quarterly, vol. 31, p. 439-465.

Urry, John (1990), The Tourist Gaze, London, Sage.

Wyckoff, Daryl D., et W. Earl Sasser (1981), The US Lodying Industry, Lexington, MA, Lexington Books. 